\author{
Using the Video Lookup Table \\ for Reflectivity Calculations: \\ Specific Techniques \\ and Graphic Results \\ Daniel H. Bass \\ Computer Science Department \\ The University of Rochester \\ Rochester, New York \\ TR51 \\ September, 1979
}

This work was supported in part by Alfred P. Sloan Foundation grant \#74-12-5 and in part by National Science Foundation grant MCS76-10825. $\mathrm{Mr}$. Bass did this work as an undergraduate student at the University of Rochester. He is presently a graduate student in the Comptuer Science Department of the University of Illinois at Urbana-Champaign.

\title{
CARLSON LIBRARY
}




\section{ABSTRACT}

Sloan and Brown [2] have shown that the video lookup table can be used to provide fast and flexible changes to a raster graphic display without changing the image memory contents. By storing encoded surface normal vectors and using the video lookup table to perform reflectivity calculations, the surface may be rapidly displayed under a wide range of lighting and reflectivity conditions.

However, there are certain drawbacks. First, quantizing the surface normals produces artifacts in the final image unlike those resulting from the usual approach of quantizing the surface brightness. Second, the lighting effects must be independent of object position, and so shadowing cannot be done with this method. Third, the process is still not quite fast enough to allow real-time rotating lights.

Results from the use of this method are shown in photographs of the display of several objects under different lighting, reflectivity conditions, and surface normal quantization schemes. 
1. INTRODUCTION

1.1 Raster Graphics

With the advent of cheap digital memory, it has become economically feasible to store a discrete version of a complex image in random-access memory, for manipulation with a computer, and for display. In this paper, we are concerned with RASTER GRAPHICS display devices. In these devices, there is an IMAGE RASTER MEMORY which stores digital, discrete image information. This information is stored in a rectangular raster of picture elements, or PIXELS. Typical raster sizes are on the order of $256 \times 256$ pixels to $1024 \times 1024$ pixels. Thus, the raster has some 256 - 1024 rows of $256-1024$ pixels in a rectangular array. A commercial TV has some 500 rows of continuous intensity information; a $512 \times 640$ raster approximates the resolution of commercial TV. Each pixel consists of a number of bits of brightness information. If there is only one bit per pixel, the pixel is either on or off (wite or black). With two bits, four brightness levels may be represented. Six bits (64 grey levels) per pixel approximates the intensity range seen on commercial black and white TV sets. Color information may be stored in the pixels as well; a color TV monitor has red, blue, and green color electron guns which, when fed different signal levels, intensify red, blue and green phosphors at different levels on the screen. These varying amounts of three primary colors combine to give the range of colors seen on the screen. If there are four bits of information for each color gun, and three guns, then $4+4+4=12$ bits are needed per pixel; $2^{4}=16$ brightnesses are possible for each primary, for a total of $2^{12}=4096$ possible colors. 
In a raster graphics display system, the image raster memory is continuously "scanned out" by electronics which converts each row of pixels to a row of intensities on a screen. The raster memory is scanned out at 30 or 60 times a second. Changing the image memory results in an immediate change on the screen; the entire image is stored explicitly in the image raster memory. In the (perhaps more familiar) vector graphics devices, a program is executed which causes electronics to paint lines on a screen according to the program. The lines so painted will quickly fade if not repainted, so a stable image only results from a quickly looping program which "refreshes" the screen at a rate above the "flicker fusion" rate (about twenty times per second). Large changes in a vector graphics image may be made very quickly (say by switching to another program wich paints something different). This is because there is no explicit version of the image to change; the image is regenerated every time by the program. In raster graphics, however, the image raster must be filled in initially to obtain an image. Such filling in may take some time, since perhaps $256 \times 512 \times 12$ (about 1.5 million) bits of information..are required. Not only could shipping this much data take time, but individually computing the intensity or color of each of some $256 \times 512$ (about 125,000) pixels can take time.

It often is useful (see Discussion section) to keep a modeled scene fixed and yet change its appearance. We may want to set up a theatrical stage and vary the lighting on it, to simulate the appearance of a building at different times of day, to experiment with different surface finishes of a solid object, etc. In "relighting," the scene is displayed under a specified configuration of lights. "Resurfacing" the objects in the scene involves the specification of a 
particular reflectance model (see below) and displaying the scene using this model. In each of these cases, traditional raster graphics practices would require re-computing the brightness or color of each pixel under the new conditions and accordingly rewriting the image memory. This paper describes the use of a facility common to most modern raster graphics devices to accomplish some "relighting" and "resurfacing" operations quickly and efficiently. The facility is the VIDEO LOOKUP TABLE, or color map (cf [11]). First, a few basics will be presented about the calculation of surface appearance under illumination.

\subsection{Reflectivity Calculations}

The following treatment is paraphrased from [2] (or see [4].)

Consider a point source of light at infinity illuminating a piece dA of a surface. The problem is to calculate how bright the surface appears from a particular viewpoint. The brightness will depend on characteristics of the light source, of the surface, and on several angles. (see Figure 1)

The light flux captured by the surface is $L \cos (I) d A$, where $L$ is the flux density ( $f l u x /$ unit area) of the source. The intensity of the surface patch is $B \cos (E) d A$, where $B$ is the luminance of the surface in the direction of the viewer. The REFLECTANCE of the surface is defined as $B / L$, and is a function of the angles $i$, and $g$.

LAMBERTIAN surfaces, also known as diffusely reflecting surfaces, have an intensity that is proportional to the cosine of e, the emittance angle; in other words, they reflect less in directions of 
low angle with respect to the surface. These surfaces have the property that they look equally bright from any direction. This is because the amount reflected per unit area decreases as the cosine of the viewing angle, but the amount of surface area seen increases as the reciprocal of the cosine of the viewing angle. Thus, the perceived brightness is independent of viewing angle, and is only determined by the illuminance, which varies as the cosine of the incidence angle.

Since reflectivity is determined by the microstructure of the surface, not all reflectivity functions are LAMBERTIAN. For example, the lunar surface reflects in all directions equally (This explains why the lunar disk does not look "rounded" like a ping-pong ball [8].) Purely SPECULAR (mirror-like) surfaces reflect only in a direction for which the angle of reflection equals the angle of incidence, and the direction of reflection and the direction of incidence are coplanar with the surface normal. Most smooth surfaces have a specular component to their reflectivity function, but in many cases some light is also reflected at angles slightly away from the predicted angle. The cosine of the angle between the predicted specular angle and the viewing angle is $2 \cos (i) \cos (e)-\cos (g)$ [6]. This quantity is unity in the correct direction and falls off to zero at pi/2 radians away from the correct direction. While actual reflectance functions are not necessarily so characterized, computer graphics workers often use the cosine of this angle as a basis for computing specular-like reflectivity functions. The sharpness of the specular peak can be increased by taking powers of the cosine. Thus, one possible reflectance formula is: 


$$
\operatorname{PHI}(I, E, G)=s(2 I E-G)^{n}+(1-s) I
$$

Where I, E, and G stand for $\cos (i), \cos (e)$, and $\cos (g) ; s$ is the fraction of specularly reflected light, and $n$ determines the sharpness of the specularity peak. 
2. METHOD

\subsection{Preview}

From the treatment of section 1.2 , it is seen that calculating the perceived brightness of a point on a surface may be solved to a first approximation if $n, s, g, i$, and $e$ are known for the point. The reflectivity formula leaves out of account such phenomena as distributed light sources, shadows, sources not at infinity, and many other effects. The parameters $n$ and $s$ may be held constant for the scene to select a particular surface finish of objects in it. $g$ is independent of surface orientation, and $i$ and $e$ are simple functions, for a fixed observer and light source, of the SURFACE NORMAL at the point of interest.

Generally, the observation wich this paper exploits is the following: if the position of the observer is held constant, as is the surface finish of the objects in the scene, then the calculation of the brightness of any point on the object may be taken to depend only on the surface normal at that point and on the lighting characteristics (direction, brightness, and color). The function giving surface brightness as a function of surface gradient is called the REFLECTANCE MAP [9]. As we have seen, such simple reflectivity models ignore important effects, but are practically very useful.

This observation is exploited in the following manner. If the scene remains static, then the surface normals do not change in time. Given a lighting situation, the appearance of each pixel will depend only on the surface normal at the point on the surface which appears in that pixel. Suppose that instead of storing surface appearance 
(intensities or colors) for each pixel in the image memory raster, representations of the surface normals are stored instead. Then all that would be needed to derive a new appearance for such a scene under different lighting would be to determine for every surface normal what the appearance would be. If the surface normals are quantized so that there are not too many of them, (say on the order of thousands), such a calculation will take little time. Then all that is needed is a way to map each normal (stored in a pixel) to its appearance on the screen.

Luckily, there is a standard piece of hardware in many raster graphics devices to accomplish this. Usually it is used to transform colors, and is called a COLOR MAP or video lookup table [11]. We use it to make the translation from surface normal to appearance automatically and continuously. "Relighting" or "resurfacing" the scene only involves rewriting the map from surface normal to appearance, and is quickly done. In the configuration used for this work, the image memory consists of 131,072 twelve-bit words, while the video lookup table has only 4096.

\subsection{The Video Lookup Table}

The video lookup table is a function which maps configurations of image memory bits into bits presented to the display (Figure 2). The table is addressed by the image memory pixel values. The contents of the table at each address is a field of bits (whose width is unrelated to the image memory pixel size. It is the table contents (not the pixel value) which determines what signals are sent to the video monitor. Thus, an image of a single bit plane has just two addresses 
in its video lookup table, one for each pixel value.

When the brightness information is stored directly in the raster, the video lookup table commonly used is the identity map, which passes the image memory contents unchanged to the monitor electron guns. Alternative maps may be used to mask, emphasize, correct for non-linear display response or select certain information stored in the raster $[11]$.

In the current University of Rochester system, the raster consists of a $256 \times 512$ matrix of 12 bit words. The black and white monitor accepts eight bits of grey-scale data per pixel, which is interpreted as a grey level from 0 to 255. The color monitor takes twelve bits of color data per pixel, which is broken down into three 4-bit words sent to the red, green and blue guns, providing 16 levels of each color. The video lookup table can map any of 4096 pixel values into 4096 possible outputs to the color guns, thus providing a perfectly general mapping capability.

\subsection{Calculating the Video Lookup Table}

Having written the normal vector information into the raster memory, to display the image requires the calculation of the reflectance map. Thus, the brigntness for all possible orientations of the surface must be calculated and stored in the proper location in the video lookup table.

In order to calculate the perceived brightness, the cosines of $i$, $e$, and $g$ (as defined previously) must be found. From linear algebra we know that for vectors $u$ and $v$, the cosine of the angle between them 
equals:

$$
\begin{gathered}
u \cdot v \\
|u| \quad|v|
\end{gathered}
$$

The vectors involved are the direction vector of the light, the normal vector and the viewing vector. The first is constant for any fixed point light source at infinity, and the second is one of the set of normal vectors that are being coded. The viewing vector is assumed always to be $(0,0,1)$ (that is, the screen is in the $X-Y$ plane, and the observer is taken to be looking along the $Z$ axis from infinitely far away). Thus a possible procedure to create a color map would be: 


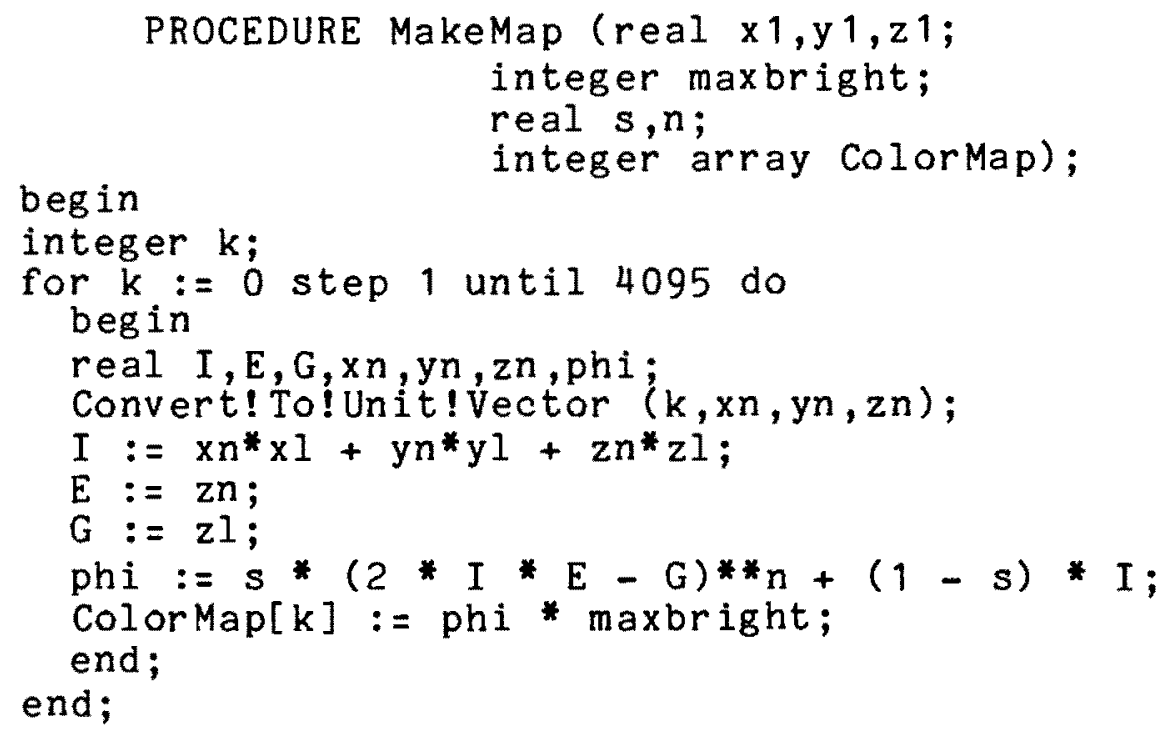

In this procedure, $(x 1, y l, z l)$ is a unit vector in the direction of the light. CONVERT!TO!UNIT!VECTOR is a procedure that finds the $k^{\text {th }}$ normal vector and places its unit components in $(x n, y n, z n)$. The other variables are the same as previously stated.

\subsection{Coding the Surface Normals}

A point on a surface is either on its visible side or on the "back" side, facing away from the viewer. When viewed from the positive $z$ direction, a surface normal is on the back side if and only if it has negative $z$ component. That is, all of the points whose vectors point away from you are hidden. Ergo, it is possible to describe all "visible vectors" as points on the hemisphere:

$$
z=\operatorname{sqrt}\left(r^{2}-x^{2}-y^{2}\right)
$$


It is first necessary to determine a set of vector equivalence classes into which all visible vectors may be grouped. Each equivalence class is represented by a approximation of those vectors in that equivalence class. Thus, wille there are an infinite number of "visible vectors," they can be classified into some finite collection of vector equivalence classes. The vectors representing the equivalence classes have a positive $z$ component, thus the additional constraint that $x^{2}+y^{2}+z^{2}=r^{2}$, for some $r$, reduces the number of independent variables to two. The two variables chosen are quantized over an appropriate range, and are in one-to-one correspondence with a set of integers $(0,1, \ldots, p)$ and $(0,1, \ldots, q)$. The equivalence classes result from the Cartesian product of the quantized variables, and the corresponding twelve-bit codes may be calculated from the Cartesian product $(0,1, \ldots, p) \times(0,1, \ldots, q)$.

One choice of independent variables is $x$ and $y$. As $x$ and $y$ both lie in the range $[-r, r]$ and there are twelve bits of memory in each pixel of the raster, six bits can be used to define $x$, and six bits can be used to define $y$. For symmetry, $x$ and $y$ are both quantized so that $p=q=62$. Choosing $r$ to be 31 causes $-31 \leq x, y \leq+31$ and so $x$ and $y$ are quantized to integers in this range. The corresponding equivalence classes are represented by vectors $(i x, i y, z)$ where ix and iy are integers, $z$ is non-negative, and $x^{2}+y^{2}+z^{2}=31^{2}$. Inversely, a twelve bit integer can be interpreted as representing a vector equivalence class that can be obtained from the following equations : 


$$
\begin{aligned}
i x & =(\text { high order } 6 \text { bits of } V)-31 \\
i y= & (\text { low order } 6 \text { bits of } V)-31 \\
z & =\text { sqrt }\left(961-x^{2}-y^{2}\right)
\end{aligned}
$$

It may be noted that while this mapping of vector equivalence classes to twelve bit codes is 1-1, it is not onto. That is, while each equivalence class is represented by a unique code, there are many codes that nave no associated vector, since the given inverse function yields an imaginary $z$ component from these codes. So by eliminating (ix,iy) points from the Cartesian product of $(-31, \ldots,+31) x$ $(-31, \ldots+31)$, where $x^{2}+y^{2}>r^{2}=961$, there remain about 3000 possible vector equivalence classes.

Care must be taken when calculating these vector equivalence classes, since the above treatment, while correct in the continuous case, is insufficient for the discrete raster case. The best approximation to the circumference of a circle, when limited to Cartesian (raster) points, often lies outside of the circle. The distance from the center to this approximated circumference of the approximation is at most $r+.5$, so the limit on $x^{2}+y^{2}$ is $r^{2}+r+$ .25 , or 992. Including these vector equivalence classes increases the number to 3125 , and this is the number of calculations needed to reevaluate the reflectance map. 
The above method, called Scheme I, based as it is on quantizing the $x$ and $y$ vector components, results in surface normals with approximately equal $x$ and $y$ coordinates being grouped into equivalence classes. This is clear in displays of spheres (Figure 3d), which show square patches of equal unit vectors. The appearance of such "quantization errors" depends on the method of groupings of surface normals into vector equivalence classes.

Other choices of independent variables give alternate codings. In scheme II, $z$ and theta are chosen, where theta $=\arctan (y / x)$. This results in dividing the "visible hemisphere" into concentric rings and pie slices. Setting $p=63$ and $q=65$ results in 4095 equivalence classes, each representing a slice from a ring. Although this method provides more equivalence classes, spheres painted with this surface normal coding appear to have a "pie-shaped" substructure, as well as suffering from a "flat top" (cf. Figure 3e). The former phenomenon is due to the theta quantization, and the latter to $z$ quantization. The $z$ quantization may be visualized in the following manner. The sphere is sliced into some number of pieces of the same thickness. The $z$ coordinate for each entire slice is assumed to be the $z$ value for the middle of that slice. The entire uppermost slice is then interpreted (wrongly) as a disk.

In Scheme III, the independent variables are phi and theta, two of the classical spherical coordinates. By replacing $z$ by phi (defined as $\arccos \left(z / \operatorname{sqr}\left(x^{2}+y^{2}+z^{2}\right)\right)$ ), the flattening of the sphere is removed (cf. Figure $3 f$ ), and the surface of the sphere is more evenly partitioned into equivalence classes. 
Scheme IV uses the fact that fewer theta intervals are needed for vectors in which phi is near zero than for those in which phi is near pi/2. Instead of having a fixed number of intervals for theta, the number of theta intervals per ring is dependant on phi. The centermost ring has three slices, the second ring has five, and the $n^{\text {th }}$ ring has $2 n+1$. For 63 rings, the total number of equivalence classes is 4095 .

Generally, any invertible mapping of surface normal vectors to twelve bit words may be used. An arbitrary look-up table with a search procedure may be used, although a function that can be solved analytically is more efficient. The choice of which mapping to use may depend on the domain of interest and psycho-physical considerations, i.e. how the result looks. 


\section{RESULTS}

The efficacy of this approach to image modification was tested in a computer program which allows various objects (currently spheres, parallelepipeds, and paraboloids of revolution) to be interactively placed in space, and their surface finishes and lighting conditions to be changed interactively.

If more than one object can be seen, the "nearer" objects might hide parts of the "farther" objects. Because the objects are convex solids, however, these hidden surfaces can be masked out easily by ordering the objects by the $z$ component of their center, and painting the farthest object first, and the nearest last. This approach is a simplified version of Roberts' [10] hidden surface algorithm, based on the assumption that all objects are either completely in front of, or behind all other objects. Writing one object over the other in the raster image memory simulates physical obscuration.

To paint a sphere of radius $r$, centered at $x 0, y 0, z 0$, the following algorithm may be used: (SHL is the left shift operator)

For all integers $x, y$ such that $x^{2}+y^{2} \leq r^{2}$ Do Begin $x$ Componentof Sur faceNormal $:=31 * x / r$ y Componentof Surface Normal $:=31 * y / r$ Raster $\left(\mathrm{x}_{0}+\mathrm{x}, \mathrm{y} 0+\mathrm{y}\right):=$ $((x$ Componentof Sur face Normal +31$)$ SHL 6$)+$ End Do (yComponentof Sur faceNormal +31 ) 
best approximation for a circle. This algorithm was extended to paint disks in which each pixel is assigned a value individually. Badler's disk drawer [10] cannot be used, since each pixel in the interior of the disk must be individually calculated and may have unique memory information. The strategem is to break up the disk into rows of pixels and step through each row; then calculate and store a value for each pixel. In a disk of integer radius $r$, there will be $2 r+1$ rows of pixels. What is needed is the extrema for each row of pixels; in other words, for each integer value of $y$, which of the points on the boundary approximation has the greatest absolute value for $x$ ?

Horn's algorithm gives the Cartesian points that form the best approximation of the first octant of a circle. Because a circle is symmetric, the entire circle, or disk boundary, can be obtained by reflecting these points. This modified algorithm paints the disks simultaneously from the "equator" out and from the "poles" in (meeting at some "latitude"). Badler's disk drawer [1] cannot be used, since each pixel in the interior of the disk must be individually calculated and may have unique memory information. In Horn's algorithm, cartesian points approximate the circumference of a circle in the first octant. Furthermore, if $(x, y)$ is such a point, then Horn claims that the next point is either $(x, y+1)$ or $(x-1, y+1)$. For each $(x, y)$ point used as the circumference in the first quadrant, the lines from $(-x, y)$ to $(x, y)$ and $\operatorname{from}(-x,-y)$ to $(x,-y)$ are encoded in the raster point by point. This paints only the middle half of the disk.

For each interior loop of Horn's algorithm (i.e. when the next point is $(x-1, y+1))$ the previous circumferential point $(x, y)$ is the end-point of a vertical line contained in the disk. This line is 
symmetric to horizontal lines in the upper and lower quarters of the disk, reflected across the diagonal. These $l$ ines are from $(-y, x)$ to $(y, x)$ and from $(-y,-x)$ to $(y,-x)$.

of course, the generation of lighting and surface effects depends only on knowing the surface normal for each point on the surface, not on the type of surface. One other useful class of solids is that of parallelepipeds. A parallelepiped may be defined by the rotation and translation of its eight corner points $( \pm \mathrm{Lx} / 2, \pm \mathrm{Ly} / 2, \pm \mathrm{Lz} / 2)$ where $L x$, Ly, and Lz are the lengths of the sides. The normals of the rotated faces are identical to the rotated normals of the faces, so by applying the same rotation transformations to the original normal vectors, the new normals may be ascertained. Those faces whose normals contain a positive $z$ component may then be orthographically projected on the screen, with the same surface normal being written into the image memory for the entire face. Once the coded surface normals are in the image memory, the video lookup table automatically insures that their appearance is consistent with current lighting and surface assumptions. The paraboloid of revolution was chosen as another example of a surface whose surface normals can easily be calculated.

So far in this paper, light has been described by intensity only, thus implying a grey-scale toning of the image. Implementation of colored lights is a minor modification [cf. 3]. First the color of the light is decomposed into component colors: red, green, and blue. The relative intensity for the light is then calculated, and the scaled red, green, and blue values are stored in three parallel arrays. The video lookup table is then computed as a combination of 
these arrays. For example: (LOR is the logical (bitwise) OR of its arguments, SHL the left shift operator)

ColorMap $(K):=(\operatorname{round}(\operatorname{rmap}(K))$ SHL 8$)$ LOR (round $(g m a p(k))$ SHL 4$)$ LOR $(\operatorname{round}(\operatorname{bmap}(K)))$

Examples of the output of this program are shown in Figure 3 . The program currently allows colored or monochromatic illumination of interactively added spheres, parallelepipeds, or paraboloids of revolution from any number of point light sources, which may be interactively added at any direction. 
4. DISCUSSION

This paper has shown a method for quickly changing the appearance of a complex scene stored in a raster graphics device by changing a video lookup table. The table translates a representation of the normal vectors to a surface to appearance. This idea has several potential uses. One usual method of visualizing a complex 3-D object on a 2-D screen is to rotate it. This can involve much computation; by using the method given here, 3-D information may be obtained by leaving the object fixed in space and rotating a light source about it. Mathematically, enough information is in two or three such views to reconstruct the 3-D structure unambiguously [7]. For this method to be applicable, the perceived brightness of a surface must be taken to depend solely upon the nature of the surface and the position of the lights.

The ability to alter the lighting and reflectivity of a static scene interactively is useful for design. The difference in time necessary to paint the image and to change its appearance when using this technique is substantial. The time to paint a single large sphere (the area being about one fourth the size of the screen) is about ten seconds, whereas the time to calculate a new video lookup table and change the appearance of the image is approximately one second.

Shadowing depends on the relative location of the objects in the screen. However, since brightness is assumed to be independent of an object's location in the screen, positional information is not encoded in the raster. Thus, shadowing cannot be accomplished with the use of a video lookup table, and these objects have quite a unique property - 
they are simultaneously transparent to the illuminating radiation (they cast no shadows) and opaque (in that the method only caters to one surface normal vector per pixel, and in that surfaces therefore have opaque-like reflectivity functions). The light source must have properties which make its effects independent of the distance of the bodies from it. In this work, we chose point sources at infinity. Other possible sources would be uniform illumination, illumination from an infinite plane, etc.

In reducing the number of calculations needed to create an image, the precision of that image is also reduced. As mentioned in section 2.4 , the continuum of surface normals is partitioned into equivalence classes (in our case, at most 4096 of them). On a surface, several adjacent points may have their differing surface normals put into the same equivalence class. This results in the surface appearing as a patchwork of areas of identical surface normal (geometrically, though not perceptually, as a strange-looking polyhedron made of plane faces joined along curved lines).

In the hardware configuration used for this work, there are only sixteen levels of brightness of each of red, green, and blue. The difference between these levels is easily detected, and so a colored sphere often appears banded. This effect swamps the above quantization errors, is inherent in the hardware, and cannot be overcome by any other shading technique. The black and white monitor has 256 levels, and so the transitions are much smoother and less noticeable. 


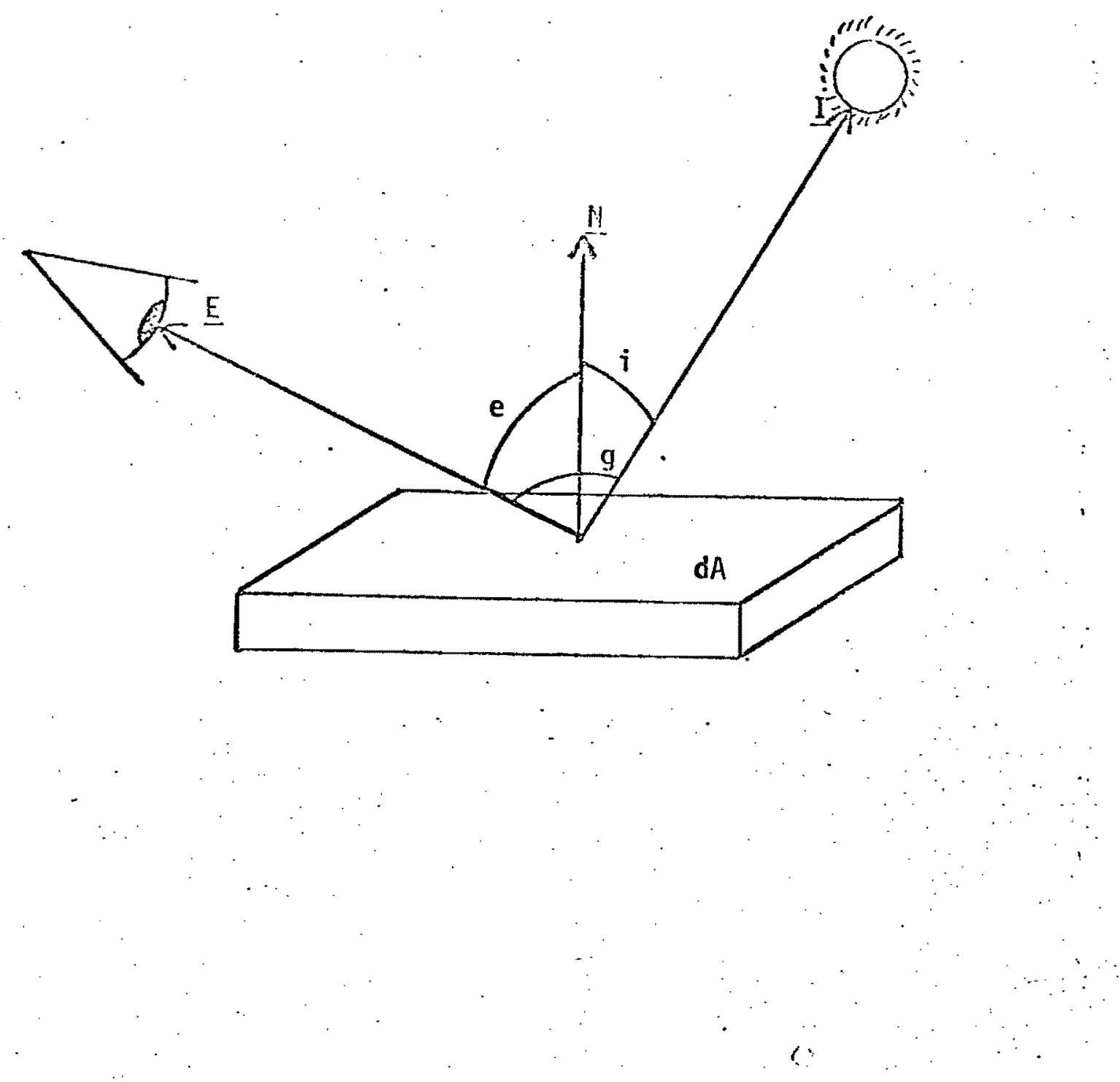

Figure 1. Reflectance Variables. A light with flux density L shines on a patch of surface dA, reflecting some component of that light in the direction of the viewer, that reflected luminance is $B$. $N$ is the unit vector normal to $d A$, and $I$ and $E$ are the unit vectors in the directions of the light source and the viewer, respectively. The incidence angle, $i$, is the angle between $N$ and $I$; similarly the emittance angle, e, is the angle between $N$ and $E$. The angle between $I$ and $E$ is called the phase angle and is deñoted $\bar{b} y$. 


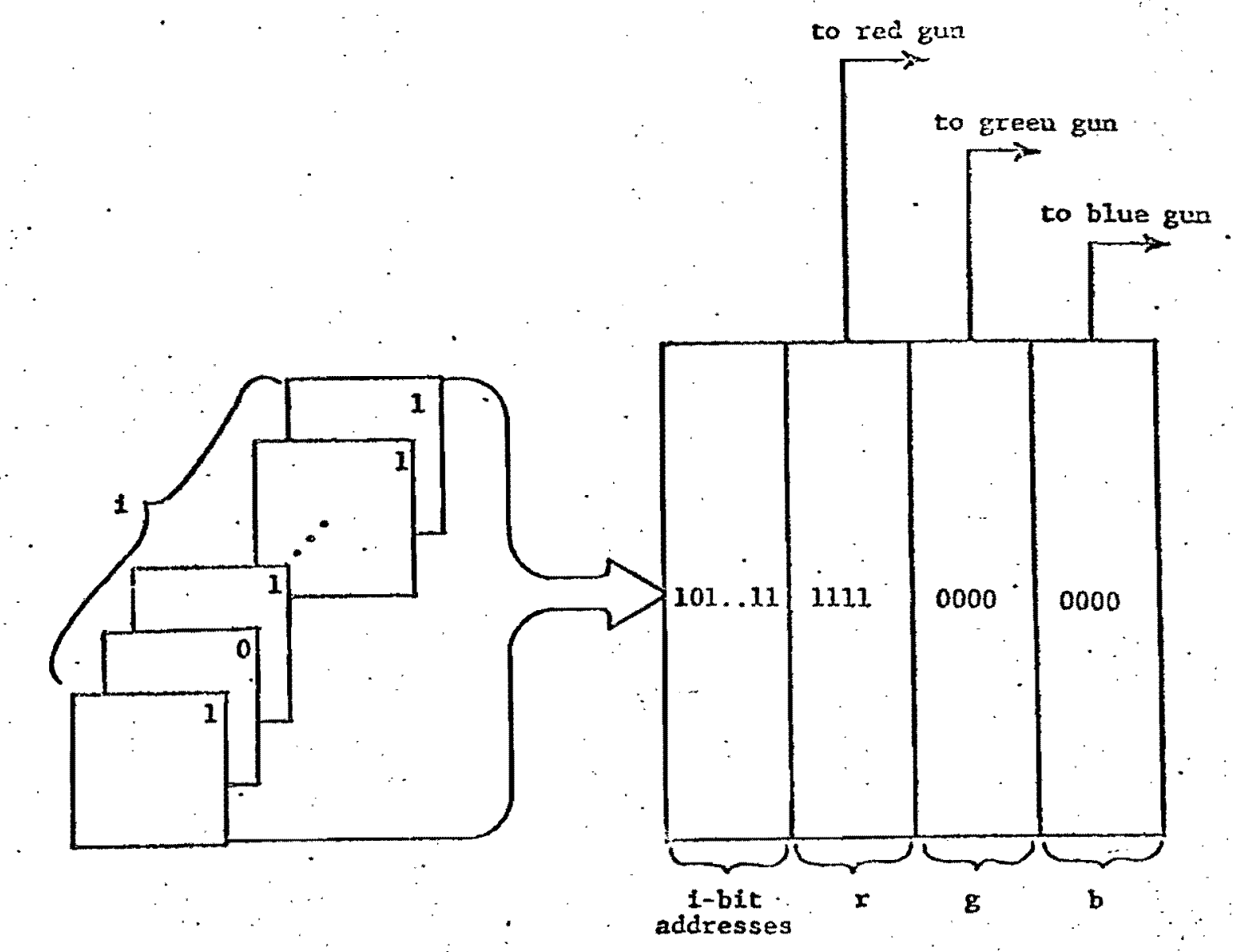

IMAGE HEMORY

COLOR MAP

Figure 2. The Video Lookup Table. An i-bit pixel in the image memory yields an i-bit index into the video lookup table. At this address in the map is found a value which is interpreted by the hardware as color or intensity for display. 


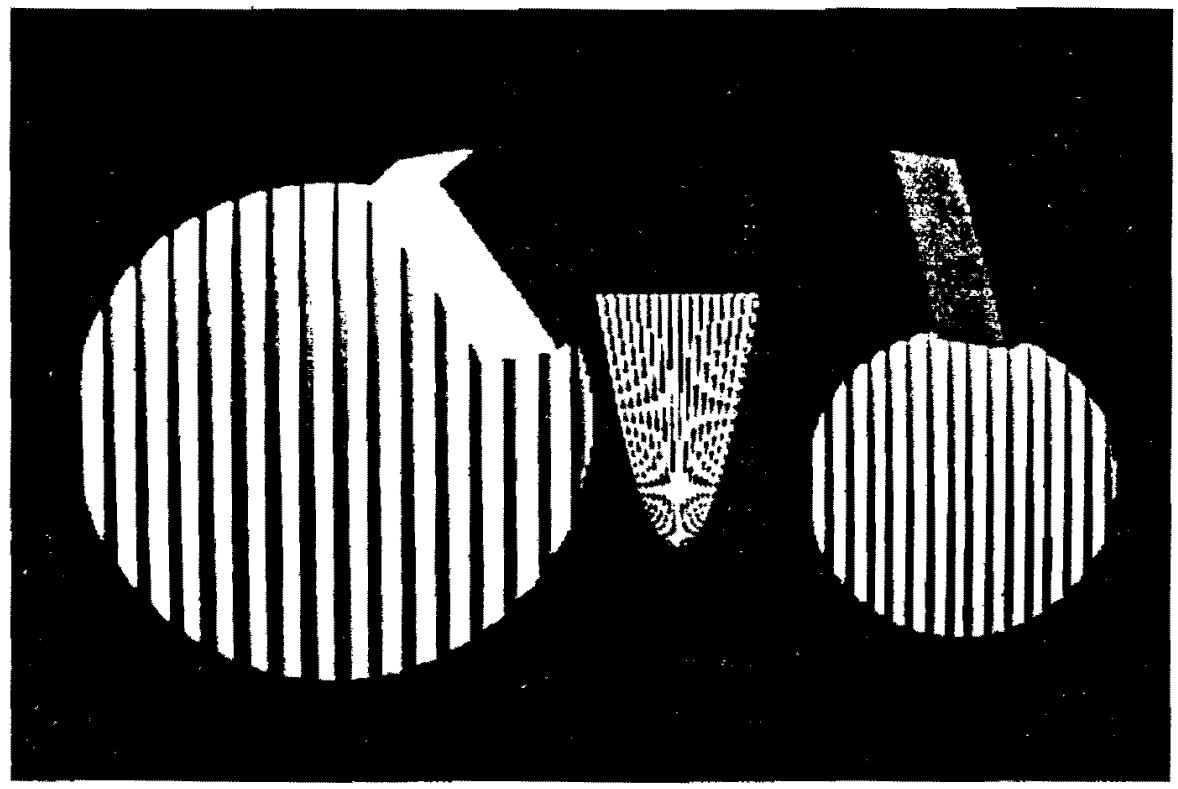

$3 a$

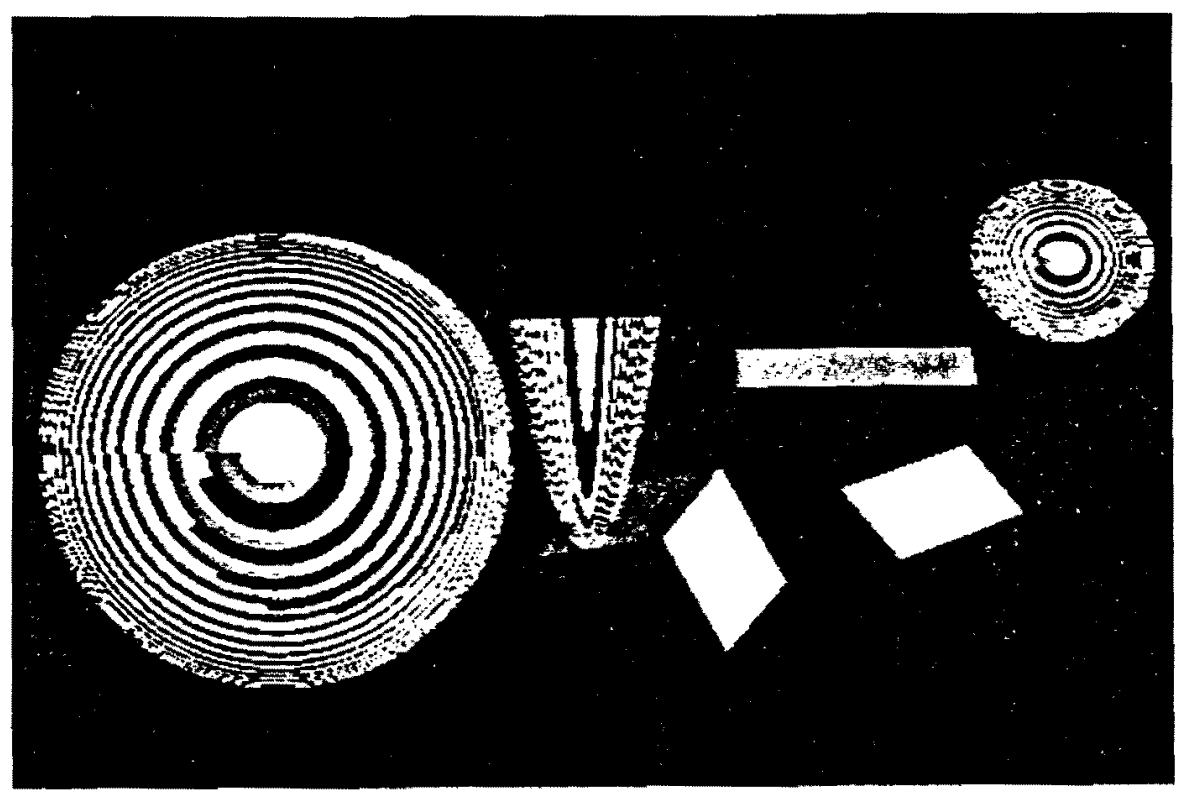

35

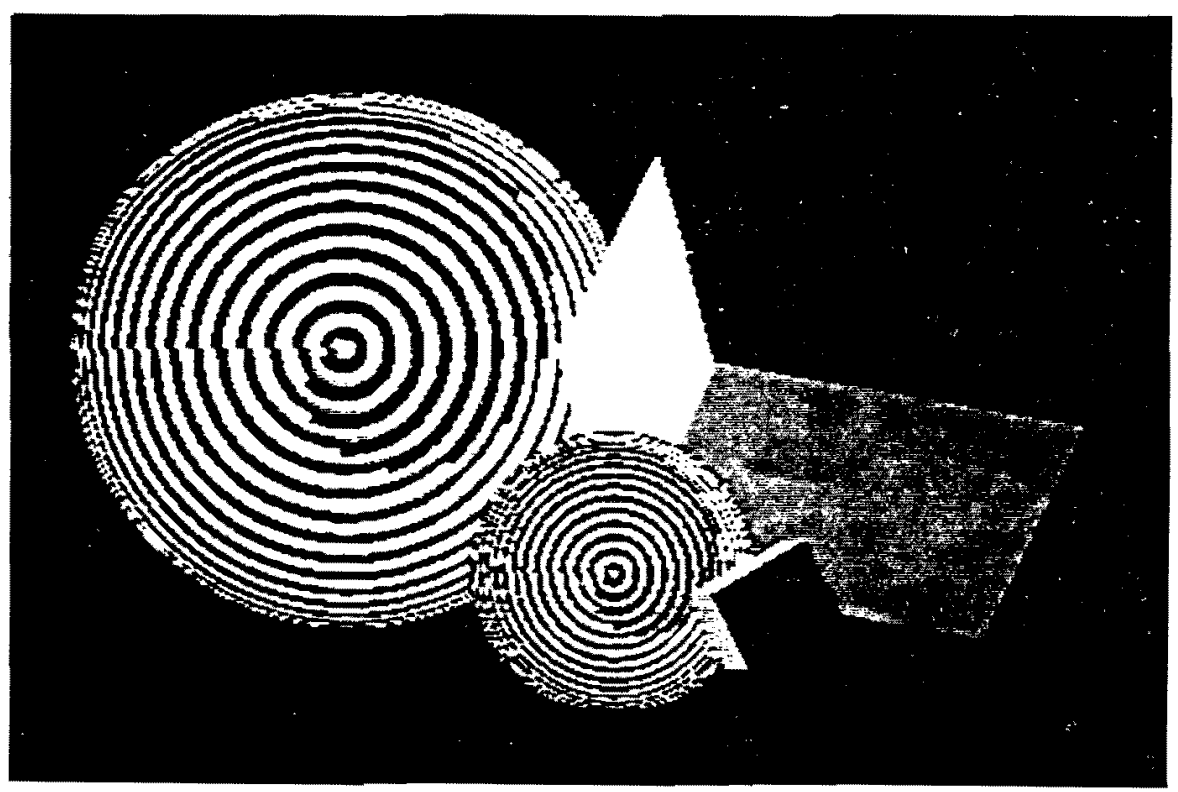



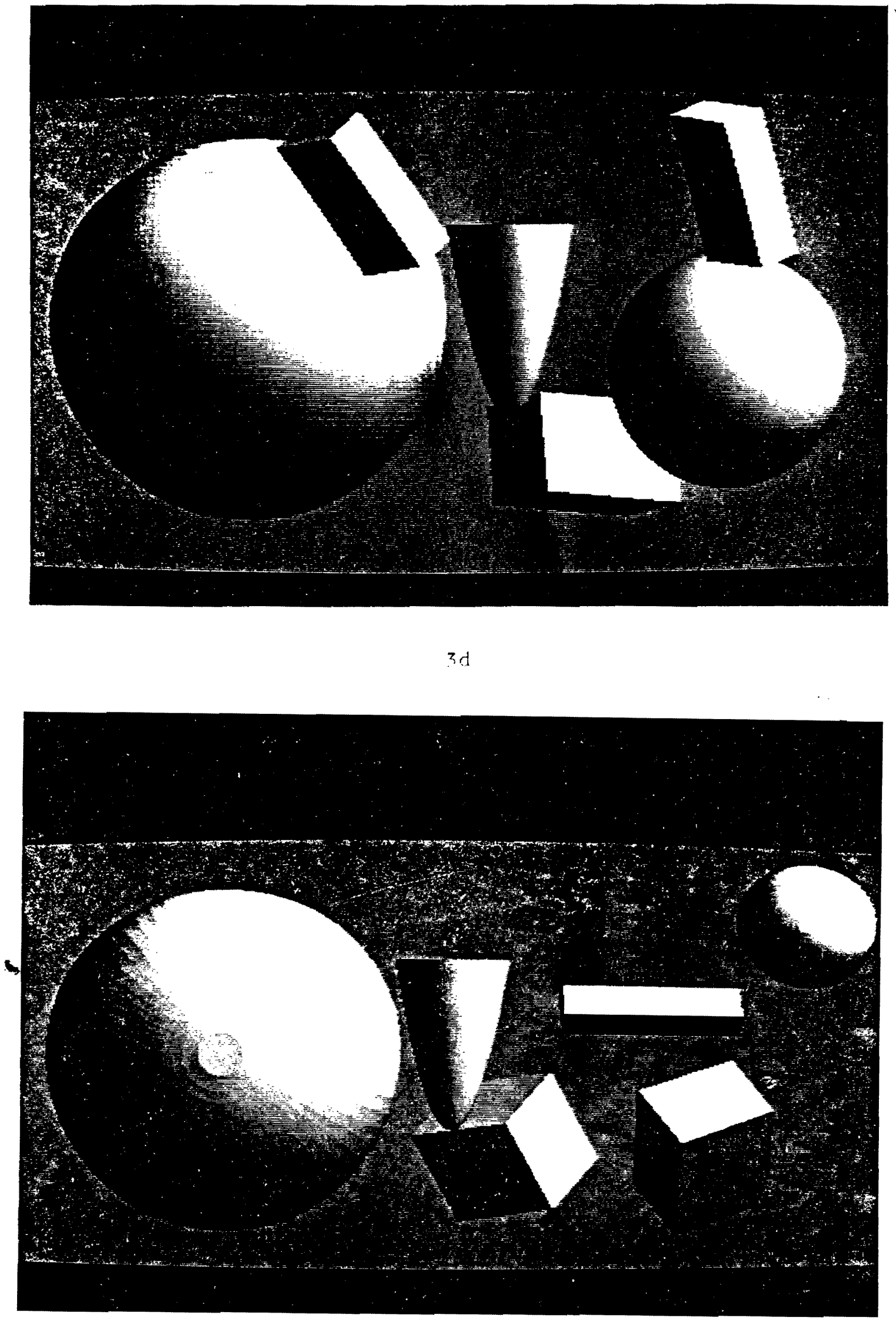


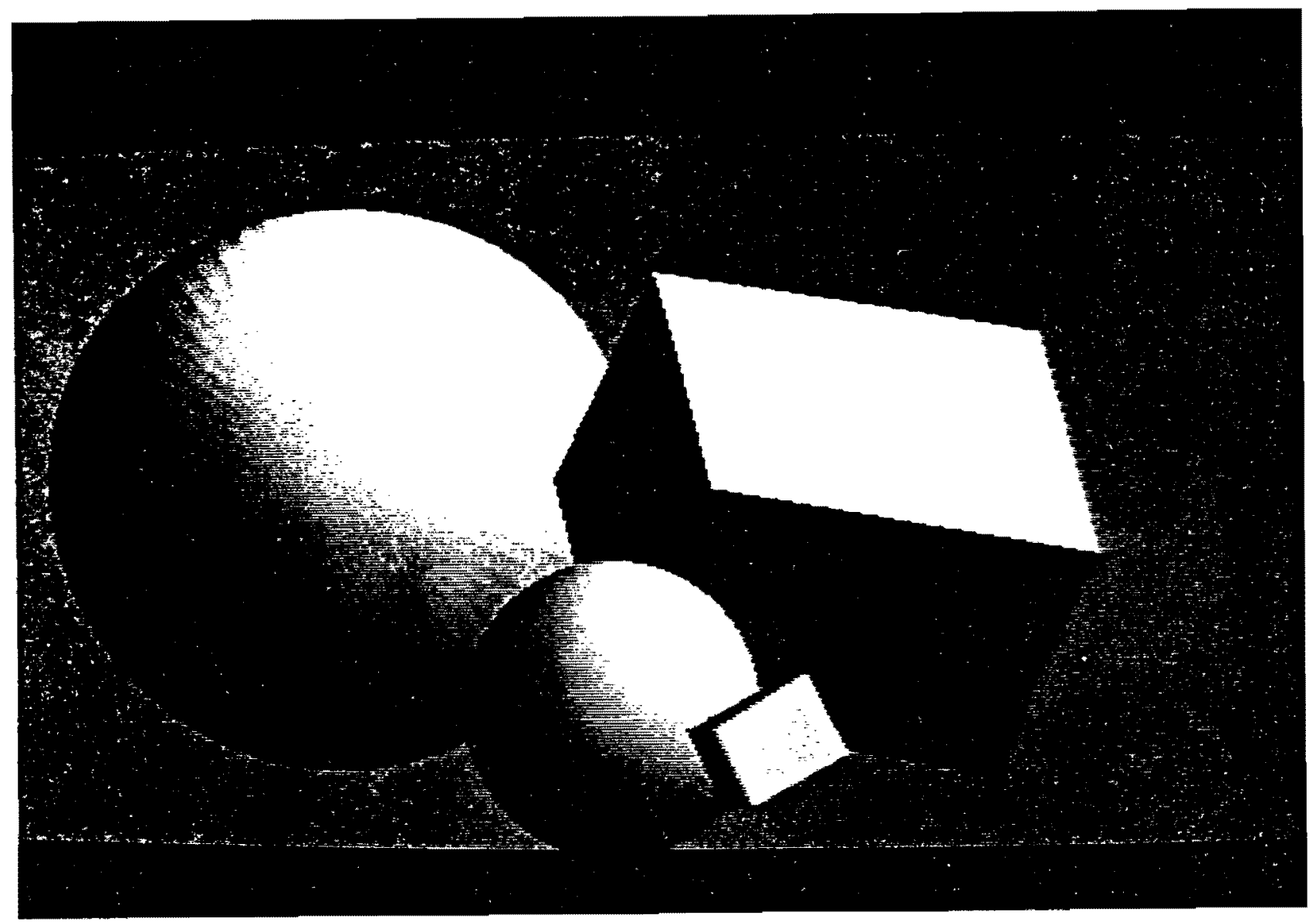

$3 f$
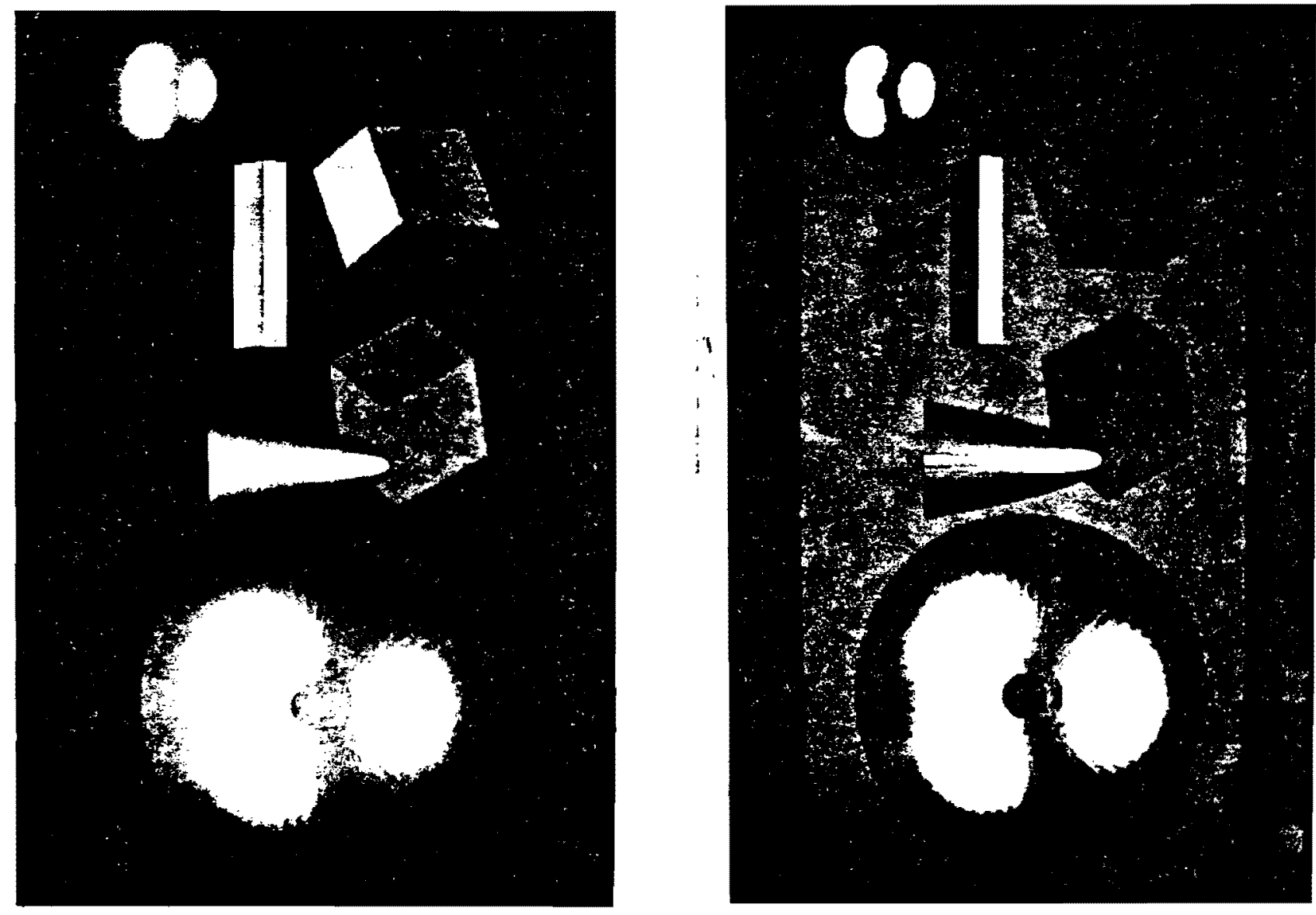
Figure 3. Results. Figures $3 a, 3 b$, and $3 c$ show the surface normals coded by schemes I, II, and III respectively. The color monitor displays some 3125 different colors for Scheme I and 4095 for Schemes II and III (one for each surface normal represented). The repeated intensities of the black and white monitor are due to the fact that the black and white monitor only snows the low-order eight bits of the image memory (the displayed intensity is the memory value modulo 256). Figures $3 d, 3 e$, and $3 f$ show the effect of a similar lighting condition on the three schemes. The varying quantization error can be seen in these photographs, and can be related to the characteristics of the coding as revealed in $3 a, 3 b$, and $3 c$. In $3 d$, the sphere exhibits square patches. In $3 \mathrm{e}$, the patches come from radial and concentric divisions. In $3 \mathrm{f}$, the concentric divisions of $3 \mathrm{e}$ are much less noticeable, particularly in the center, although the radial quantization is still quite apparent. Figures $3 g$ and $3 h$ show objects coded with scheme II with differing surface characteristics. In $3 \mathrm{~g}$, the reflectivity of the objects is $65 \%$ specular and in 3 , the reflectivity is $95 \%$ specular. 


\section{REFERENCES}

1. D.H. Ballard and C.M. Brown, Computer Vision, in progress.

2. K.R. Sloan and C.M. Brown, "Color Map Techniques", University of Rochester Computer Science Dept. TR36, October 1978; Computer Graphics and Image Processing 10, 4, Aug., 1977, pp. $297-317$.

3. B.K.P. Horn, "Using Synthetic Images to Register Real Images with Surface Models", Communications of the ACM, 21 \#11, November 1978.

4. B.K.P. Horn, "Circle Generators for Display Devices", $\frac{\text { Computer }}{280-288 .}$ Graphics and Image Processing 5, 1976, pp

5. B.K.P. Horn, R.J. Woodham, \& W. Silver, Determining Shape and Reflectance Using Multiple Images, AI Memo 490, 1978, AI Lab, MIT.

6. Computer Science and Computer Engineering Research Review, University of Rochester, 1978-1979.

7. B.K.P. Horn, The Psychology of Computer Vision, (Patrick H. Winston, Ed.), McGraw-Hill, New York 1975, pp 137-138.

8. B.K.P. Horn, Image Intensity Understanding, AI Memo 335, 1975, AI Lab, MIT.

9. L.G. Roberts "Machine Perception of Three-Dimensional Solids", MIT Lincoln Laboratory, TR 315, 1963; Optical and Electro-Optical Information Processing (Tipper et a1., Eds.) MIT Press 1963.

10. N.I. Badler, "Disk generators for a raster display device", Computer Graphics and Image Processing, 6, 1977, pp. 589-593.

11. B.K.P. Horn and R. W. Sjoberg, "Calculating the Reflectance Map" Applied Optics, to appear. 


\section{ACKNOWLEDGEMENTS}

This work was supported in part by Alfred P. Sloan Foundation grant 74-12-5, in part by National Science Foundation grant MCS76-10825, and in part by the Computer Science Department of the University of Rocnester.

I would like to thank Cnristopner Brown for nis guidance and many nelpful suggestions ine made in the writing of the paper. This work originated as a class project in an undergraduate computer grapnics course at the University of Rochester. Kennetin Sloan developed Scineme III and suggested the independence of the scnemes from tine video lookup table technique. 